\title{
Non-Local Finite-Size Effects in the Dimer Model ${ }^{\star}$
}

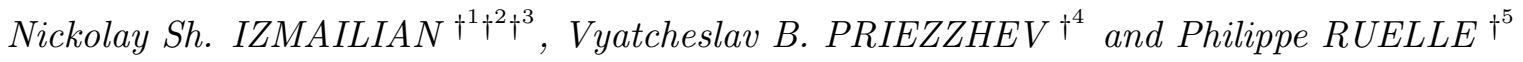 \\ $\dagger^{1}$ Institute of Physics, Academia Sinica, Nankang, Taipei 11529, Taiwan \\ E-mail: izmailan@phys.sinica.edu.tw \\ $\dagger^{2}$ Yerevan Physics Institute, Alikhanian Brothers 2, 375036 Yerevan, Armenia \\ $\dagger^{3}$ National Center of Theoretical Sciences at Taipei, Physics Division, \\ National Taiwan University, Taipei 10617, Taiwan \\ $\dagger^{4}$ Bogolyubov Laboratory of Theoretical Physics, Joint Institute for Nuclear Research, \\ 141980 Dubna, Russia \\ E-mail: priezzvb@theor.jinr.ru \\ $\dagger^{5}$ Institut de Physique Théorique, Université catholique de Louvain, \\ 1348 Louvain-La-Neuve, Belgium \\ E-mail: ruelle@fyma.ucl.ac.be
}

Received September 29, 2006, in final form December 12, 2006; Published online January 04, 2007

Original article is available at http://www.emis.de/journals/SIGMA/2007/001/

\begin{abstract}
We study the finite-size corrections of the dimer model on $\infty \times N$ square lattice with two different boundary conditions: free and periodic. We find that the finite-size corrections depend in a crucial way on the parity of $N$, and show that, because of certain non-local features present in the model, a change of parity of $N$ induces a change of boundary condition. Taking a careful account of this, these unusual finite-size behaviours can be fully explained in the framework of the $c=-2$ logarithmic conformal field theory.
\end{abstract}

Key words: dimer model; finite-size corrections; conformal field theory

2000 Mathematics Subject Classification: 82B20; 81T40

\section{Introduction}

The dimer model is extremely simple to define. We take a rectangular grid $\mathcal{L}$ with $M$ rows and $N$ columns, and consider all arrangements of dimers (dominoes) so that all sites of $\mathcal{L}$ are covered by exactly one dimer. An example of such an arrangement for a $9 \times 18$ grid is shown in Fig. 1a. In case both $M$ and $N$ are odd, one site has to be removed from the grid; this site is conventionally to be one of the four corners.

One can be more general and introduce monomers (vacancies), namely fixed sites which cannot be covered by dimers. A dimer arrangement is possible in presence of $m$ monomers provided the numbers of sites which must be covered by dimers and belonging to the even sublattice and to the odd sublattice (sum of coordinates even or odd resp.) are equal. In particular, $M N-m$ must be even. A dimer configuration on a $9 \times 18$ grid with four monomers is shown in Fig. 1b. The case $m=0$ (or $m=1$ if $M N$ is odd) is referred to as the close-packed limit of the dimer model. Corresponding to these arrangements of monomers and dimers, we

${ }^{\star}$ This paper is a contribution to the Proceedings of the O'Raifeartaigh Symposium on Non-Perturbative and Symmetry Methods in Field Theory (June 22-24, 2006, Budapest, Hungary). The full collection is available at http://www.emis.de/journals/SIGMA/LOR2006.html 

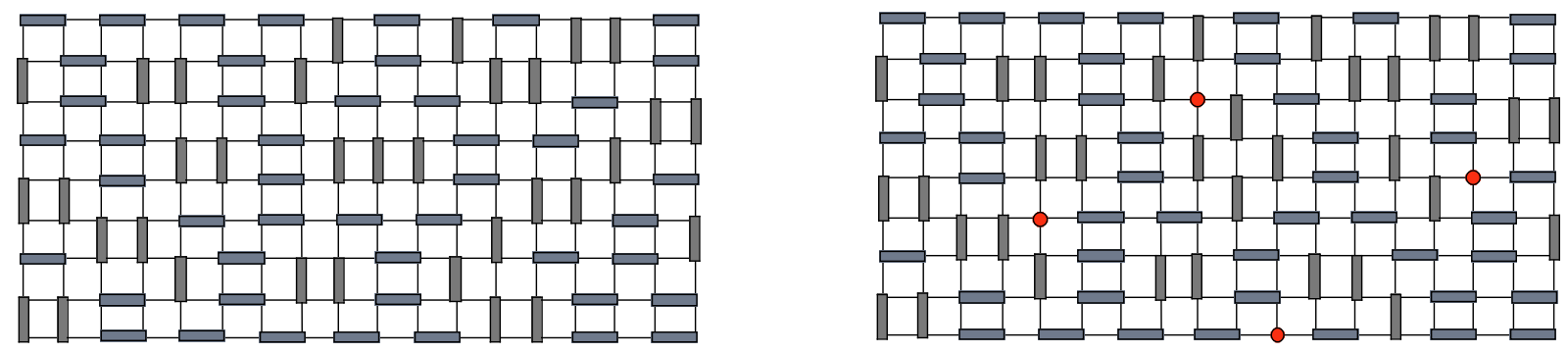

Figure 1. (a) The left figure shows one among some $4.653 \times 10^{18}$ close-packed dimer coverings on a $9 \times 18$ grid. (b) The right figure shows a dimer configuration on the same grid but with four monomers, represented by red dots. The insertion of the four monomers has reduced the number of configurations by a factor roughly equal to 57.5 , down to $8.097 \times 10^{16}$.

introduce the partition function

$$
Z\left(x, y \mid z_{1}, \ldots, z_{m}\right)=\sum_{\text {coverings }} x^{n_{h}} y^{n_{v}}
$$

It counts the number of dimer coverings in presence of $m$ monomers located at positions $z_{1}, \ldots, z_{m}$, in the bulk or on boundaries, with respective weights $x$ and $y$ assigned to horizontal and vertical dimers. As the number $n_{h}+n_{v}$ of dimers is fixed, the partition function essentially depends on $x, y$ through the ratio $x / y$ only

There are obvious generalizations (other types of lattices, coverings by other lattice animals), but the basic questions remain the same. Depending on the values of the parameters (here, $x / y)$, can the system become critical and does it exhibit phase transitions? What is the probability that certain chosen bonds be covered by dimers? How do monomers affect the number of dimer coverings? More importantly for our purpose here, what are the finite-size correction terms in the partition function, and, in case the system is critical, is its scaling regime conformally invariant?

The dimer model has been originally introduced to model physical adsorption of diatomic molecules on crystal surfaces [1]. The first studies of the dimer model were conducted in the early sixties, with pioneering works by Kasteleyn [2, 3], Fisher [4], and Fisher and Temperley [5]. Soon after that, correlations between dimers and monomers on the square lattice have been studied in [6, 7], and have been revisited more recently for the triangular lattice in [8,9]. The effects caused by the insertion of monomers have also been reconsidered recently [10, 11, 12]. Finite-size effects also have a long history, starting in [4, 13], with many subsequent works $[14,15,16,17,18,19,20,21,11]$.

Many critical systems have been shown to have a local scale invariance, so that their scaling limit can be described by a conformal field theory. Such a theory is primarily (but nonexclusively) parametrized by the value of its central charge $c$, which itself is related to the finite-size corrections to the critical free energy.

The calculation of the central charge based on the finite-size corrections has led to some confusion in the literature. Indeed it is well-known since [13] that the finite-size corrections depend on the parities of $M$ and $N$. This has prompted several authors to claim that correspondingly the central charge was also dependent of the parities of $M$ and $N$ [10].

Here we reanalyze this question. The finite-size corrections determine univoquely the effective central charge $c_{\text {eff }}=c-24 h_{\text {min }}$ [22]. To extract the central charge itself, one needs to determine the groundstate energy $h_{\min }$ of the Hamiltonian with prescribed boundary conditions. So because of $h_{\text {min }}$ the effective central charge depends on the boundary conditions. We show, by changing variables from dimer coverings to spanning trees (and more generally, to arrow configurations), 
that a change of parity of $M$ or $N$ has precisely the effect of changing the boundary conditions. The known values of $h_{\mathrm{min}}$ and the values of $c_{\text {eff }}$ as computed from the finite-size corrections enable us to obtain a consistent value for the central charge, here equal to $c=-2$. Surprisingly, this is not the only consistent value of $c$ that can be used to describe the dimer model, since it has been shown in that a dimer configuration can be encoded in a height function [23], which converges in the scaling limit to a Gaussian field, with $c=1$ [24]. We will discuss this peculiar situation in the Conclusion.

In what follows, we consider the dimer model on a square lattice in the close-packed limit, and for two different boundary conditions, free and periodic. In Section 2, we collect the known results for the finite-size corrections, and the way these relate to the central charge. In Sections 3 and 4 , we compute the central charge from the finite-size analysis respectively on a strip with free boundary conditions, and on a cylinder (periodic boundary conditions). The last section summarizes our point of view on the description in terms of $c=-2$ versus the one based on $c=1$, relating this issue to the more general monomer-dimer problem. The present results have been reported in [21], but we take here the opportunity to give more details.

\section{$2 \quad$ Finite-size analysis}

The partition function (1), in the close-packed case, has been first computed by Kasteleyn as the Pfaffian of a certain matrix [2]. Since then it has reproduced by a variety of methods [25], including that based on spanning trees $[26,27]$ which we will use later on.

The partition function, up to an irrelevant factor, depends on $x / y$ only. In the infinite volume limit, the dimer model on the square lattice becomes critical but does not show a phase transition (unlike on other lattices). The critical properties do not depend on the values of $x / y$ (provided it is neither zero nor infinite), so we set $x=y=1$ in the following. The partition function,

$$
Z(M, N)=\# \text { dimer coverings, }
$$

simply counts the number of ways the grid $\mathcal{L}$ (minus a corner if $M N$ is odd) can be fully covered by dimers. The topology of $\mathcal{L}$ is fixed by the boundary conditions: it forms a rectangle if free boundary conditions are imposed in two directions (like in Fig. 1a), or a cylinder if periodic boundary condition is chosen in the horizontal direction.

We are especially interested in the free energy $F_{N}$ per unit of height for an $\infty \times N$ lattice,

$$
F_{N}=-\lim _{M \rightarrow \infty} \frac{1}{M} \log Z(M, N) .
$$

The free energy depends on the boundary conditions, free or periodic, and on the parity of $N$. This leads to four quantities $F_{N \text {,even }}^{\text {free }}, F_{N \text {,odd }}^{\text {free }}, F_{N \text {,even }}^{\text {per }}$ and $F_{N, \text { odd }}^{\text {per }}$.

The partition functions with these boundary conditions can be expressed in terms of $Z_{\alpha, \beta}$ for $\alpha, \beta=0, \frac{1}{2}[20]$, with

$$
Z_{\alpha, \beta}^{2}(1, M, N)=\prod_{n=0}^{N-1} \prod_{m=0}^{M-1} 4\left[\sin ^{2} \frac{\pi(n+\alpha)}{N}+\sin ^{2} \frac{\pi(m+\beta)}{M}\right] .
$$

The full expansion of $Z_{\alpha, \beta}$ for large $M, N$ has been obtained in [19]. Using these results, one can easily obtain the dominant finite-size corrections terms for the above four free energy densities, as power series in $N$.

For an infinitely long strip of width $N$ with free boundary conditions, one obtains

$$
F_{N, \text { odd }}^{\text {free }}=-\frac{G}{\pi} N-\frac{G}{\pi}+\frac{1}{2} \log (1+\sqrt{2})+\frac{\pi}{12} \frac{1}{N}+\cdots
$$




$$
F_{N, \text { even }}^{\text {free }}=-\frac{G}{\pi} N-\frac{G}{\pi}+\frac{1}{2} \log (1+\sqrt{2})-\frac{\pi}{24} \frac{1}{N}+\cdots,
$$

where $G=0.915965$ is the Catalan constant.

The analogous results for the periodic case, i.e. an infinite cylinder of perimeter $N$, read [13]

$$
F_{N, \text { odd }}^{\text {per }}=-\frac{G}{\pi} N+\frac{\pi}{12} \frac{1}{N}+\cdots, \quad F_{N, \text { even }}^{\text {per }}=-\frac{G}{\pi} N-\frac{\pi}{6} \frac{1}{N}+\cdots .
$$

The dominant term in these expressions yields the exponential growth of the number of dimer coverings of a $M \times N$ grid, namely $Z(M, N) \simeq\left(e^{G / \pi}\right)^{M N}=(1.79162)^{M N}$, for both types of boundary conditions.

The general form of the free energy per unit length of an infinitely long strip of finite width $N$ at criticality is

$$
F_{N}=f_{\text {bulk }} N+2 f_{\text {surf }}+\frac{A}{N}+\cdots
$$

where $f_{\text {bulk }}$ is the free energy per bulk site, and $f_{\text {bulk }}+f_{\text {surf }}$ is the free energy per boundary site (assuming identical boundary conditions on the two sides of the strip), and $A$ is a constant. Unlike the free energy densities $f_{\text {bulk }}$ and $f_{\text {surf }}$, the constant $A$ is universal. The value of $A$ is related to the central charge $c$ of the underlying conformal theory, and depends on the boundary conditions in the transversal direction. The result is that $A$ is proportional to the effective central charge $c_{\text {eff }}=c-24 h_{\min }[28,29,30]$,

$$
\begin{aligned}
& A=-\frac{\pi}{24} c_{\text {eff }}=\pi\left(h_{\mathrm{min}}-\frac{c}{24}\right) \text { on a strip, } \\
& A=-\frac{\pi}{6} c_{\mathrm{eff}}=4 \pi\left(h_{\mathrm{min}}-\frac{c}{24}\right) \text { on a cylinder. }
\end{aligned}
$$

The number $h_{\min }$ is the smallest conformal weight in the spectrum of the Hamiltonian with the given boundary conditions (for the cylinder, we assumed that this operator is scalar, $h_{\min }=$ $\left.\bar{h}_{\min }\right)$.

The values of $A$ given above are most easily understood from the transfer matrix formalism for calculating the lattice partition function, even though the result seems generally more valid (i.e. when there is no transfer matrix). Let us first consider a cylinder of perimeter $N$ and height $M$, with certain boundary conditions $|b\rangle$ and $|t\rangle$ on the bottom and top edges ${ }^{1}$. The transfer matrix $\mathcal{T}_{\text {cyl }}$ is labelled by the degrees of freedom living on a horizontal circle, in terms of which the partition function is equal to $Z(M, N)=\left\langle b\left|\mathcal{T}_{\text {cyl }}^{M}\right| t\right\rangle$. In the thermodynamic limit, the vector space spanned by the degrees of freedom living on a circle (space coordinate) goes over to an infinite Hilbert space $\mathcal{H}_{\text {cyl }}$, and the transfer matrix, which can be viewed as a unit translation operator in the vertical direction (time coordinate), can be written as $\mathcal{T}_{\text {cyl }}=\mathrm{e}^{-H_{\text {cyl }}}$, in terms of a Hamiltonian $H_{\text {cyl }}$. In the large $M$ limit, the partition function $Z(M, N)=\langle b| \mathrm{e}^{-M H_{\text {cyl }}|t\rangle}$ will be dominated by ground-state $E_{0}$ of $H_{\text {cyl }}$, so that $F_{N}=-\lim _{M \rightarrow \infty} \frac{1}{M} \log Z(M, N)=E_{0}$.

The Hamiltonian is the charge associated to the time-time component of the stress-energy tensor $H_{\mathrm{cyl}}=\frac{1}{2 \pi} \int_{0}^{N} \mathrm{~d} u T_{00}$. If the system is critical and conformally invariant, the Fourier modes of the stress-energy tensor are the left and right Virasoro modes $L_{n}, \bar{L}_{n}$, and the Hamiltonian is simply given by the zero-th moded Virasoro generators as $H_{\text {cyl }}=\frac{2 \pi}{N}\left(L_{0}+\bar{L}_{0}-\frac{c}{12}\right)$. This last formula assumes a normalization where the ground-state energy vanishes in the thermodynamic

\footnotetext{
${ }^{1}$ In the limit $M \rightarrow \infty$, we expect that the specific boundary conditions we choose do not matter if the theory is local, since the boundaries are sent off to infinity. We could as well choose periodic boundary condition in the vertical direction, closing the cylinder into a torus.
} 
limit $N \rightarrow \infty$, and therefore ignores the bulk term in (5) (in the periodic geometry we consider here, there is no surface and therefore no surface term in (5)). In addition the Hilbert space decomposes into representations of the left and right Virasoro algebras as $\mathcal{H}_{\text {cyl }}=\oplus_{h, \bar{h}} N_{h, \bar{h}} \mathcal{R}_{h} \otimes$ $\mathcal{R}_{\bar{h}}$, in which the $N_{h, \bar{h}}$ are integer multiplicities. The representations $\mathcal{R}_{h} \otimes \mathcal{R}_{\bar{h}}$ are highest weight representations, meaning that they are built from a highest weight state $|h\rangle \otimes|\bar{h}\rangle$ by applying all Virasoro modes. All states of the representation are eigenvectors of $L_{0}+\bar{L}_{0}$, but the state with the smallest eigenvalue is the highest weight state, $\left(L_{0}+\bar{L}_{0}-h-\bar{h}\right)|h\rangle \otimes|\bar{h}\rangle=0$. Putting all together, one obtains that the ground-state of $H_{\text {cyl }}$ in $\mathcal{H}_{\text {cyl }}$ is equal to

$$
E_{0}=\frac{2 \pi}{N}\left(h_{\min }+\bar{h}_{\min }-\frac{c}{12}\right),
$$

where $h_{\text {min }}$ and $\bar{h}_{\text {min }}$ label the representation in $\mathcal{H}_{\text {cyl }}$ with the smallest $\left(L_{0}+\bar{L}_{0}\right)$ eigenvalue. Assuming the equality $h_{\min }=\bar{h}_{\text {min }}$ yields the result quoted above.

For the strip, a first change is that one has to specify the boundary conditions $a, b$ on the edges on the strip. The lattice transfer matrix depends on $a, b$, as do the Hilbert space $\mathcal{H}_{a, b}$ and the Hamitonian $H_{a, b}$ in the thermodynamic limit, but the formula $F_{N}=E_{0}^{a, b}$ still holds.

The second change is that there is only one copy of the Virasoro algebra on the strip. It implies that the Hamiltonian is now equal to $H_{a, b}=\frac{\pi}{N}\left(L_{0}-\frac{c}{24}\right)$, and the Hilbert space decomposition reads $\mathcal{H}_{a, b}=\oplus_{h} N_{h}^{a, b} \mathcal{R}_{h}$, leading directly to the value of $A$ given in (6). Again the normalization means that the quantum Hamiltonian ignores the bulk and surface terms in (5).

The finite-size corrections computed above for $F^{\text {free }}$ and $F^{\text {per }}$ have the correct form (5), but at first sight look paradoxical. Comparing them with (6) and (7), we see that the effective central charge depends on the parity of $N$. The boundary conditions do not seem to change with the parity of $N$. This would imply that $h_{\text {min }}$ does not change either, that the central charge itself has to change. This would be most peculiar since the central charge also controls the bulk conformal theory.

The only way out is to accept that the boundary conditions change with the parity of $N$, although this is not apparent in the dimer variables. We will show in the following that the effective central charge changes with the parity of $N, c_{\text {eff }}=-2$ for $N$ odd and $c_{\text {eff }}=1$ for $N$ even, not because the central charge changes, but because the value of $h_{\min }$ changes, due to the fact that a change in the parity of $N$ effectively changes the boundary condition. On the strip, this effect has been already noted in [31].

To understand this peculiarity of the dimer model, we consider a change of variables, namely we replace dimer configurations by arrow configurations on a sublattice. On the strip, the arrow configurations define spanning trees, so that the dimer model is mapped to the spanning tree model [27, 31] or, equivalently, the Abelian sandpile model [32]. The case of the cylinder is slightly more complicated because the arrow configurations do not always define spanning trees. The analysis of this case is however similar.

\section{Dimers on a strip}

Let us consider first the dimer model on the rectangular lattice $\mathcal{L}$ of size $M \times N$ with free boundary conditions. Since we are interested in the limit $M \rightarrow \infty$, the parity of $M$ will not matter here. For simplicity, we take $M$ odd, and discuss successively the cases $N$ odd and $N$ even.

When $M$ and $N$ are both odd, there is a well-known bijection [26] between close-packed dimer coverings of $\mathcal{L}$ with one corner removed and spanning trees on the odd-odd sublattice $\mathcal{G} \subset \mathcal{L}$ (thus $\mathcal{G}$ contains the sites whose coordinates, counted from the lower left corner, are both odd).

A dimer containing a site of $\mathcal{G}$, in red in Fig. 2, can be represented as an arrow directed along the dimer from this site to a nearest neighbour site in $\mathcal{G}$. It is easy to prove that the resulting 

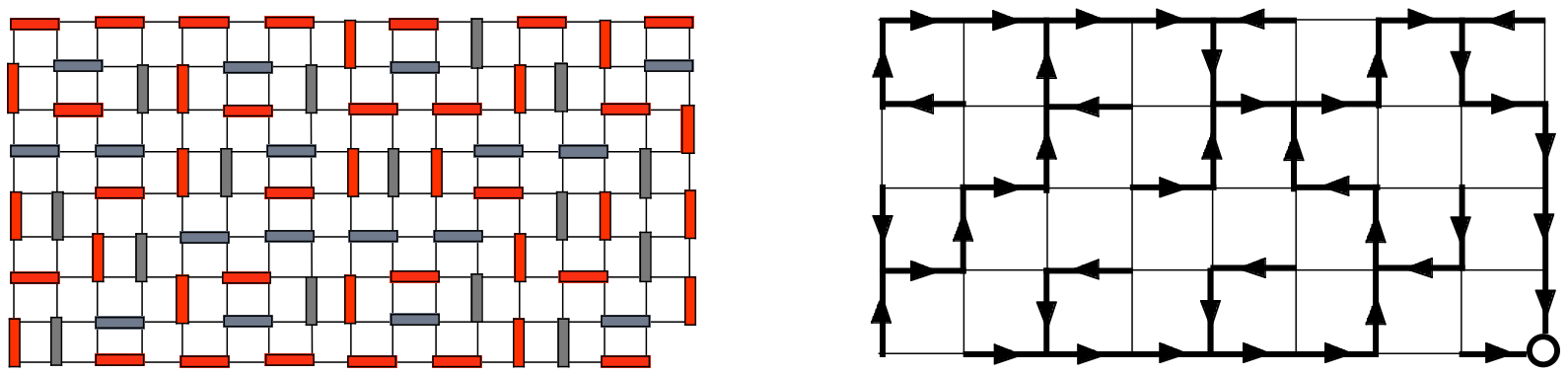

Figure 2. The left figure represents a dimer covering of a $9 \times 17$ lattice. The red dimers are those which touch the odd-odd sublattice $\mathcal{G}$. The right figure shows the corresponding spanning tree on the $5 \times 9$ lattice $\mathcal{G}$, with a unique root pictured by an open dot.

set of arrows generates a uniquely defined spanning tree; all the arrows point to a unique root, located at the corner which had been removed from $\mathcal{L}$ (see Fig. 2). Since the dimers which do not contain a site of $\mathcal{G}$ are completely fixed by the others, one has a one-to-one correspondence between dimer coverings on $\mathcal{L}$ minus a corner and spanning trees on $\mathcal{G}$, rooted at the removed corner. The Kirchhoff theorem then expresses the number of dimer configurations as $Z=\operatorname{det} \Delta$, where $\Delta$ is the Laplacian matrix on $\mathcal{G}$ with appropriate boundary conditions, see Section IV for a proof of this result. Viewing $\mathcal{G}$ as a graph, one sets $\Delta_{i j}=-1$ if sites $i$ and $j$ are connected in $\mathcal{G}$, and $\Delta_{i i}$ is equal to the number of sites in $\mathcal{G}$ that $i$ is connected to, plus 1 if $i$ is connected to the root. As shown in [32], spanning trees on $\mathcal{G}$, rooted at a corner, are in bijection with the configurations of the Abelian sandpile model (ASM) on $\mathcal{G}$, with closed boundary conditions on the four boundaries, the only sink (dissipative) site being the root of the trees.

When $M \rightarrow \infty$, the lattice $\mathcal{G}$ becomes an infinitely long strip of width $N$. The root is sent to infinity so that no boundary site (at finite distance) is connected to the root. In the ASM language, this means that the boundaries are not dissipative and hence there is no out-flow of sand at the boundaries; such boundaries conditions have been called closed boundary conditions.

There are now very strong arguments to believe that the (scaling limit of the) ASM on a square lattice is described by a logarithmic conformal field theory with a central charge $c=-2$ $[32,33,34,35,36]$. In particular, the spectrum of the ASM Hamiltonian on a slice of the strip with closed boundary conditions at the two ends has been computed in [33]. There are two ground-states, the identity operator and its logarithmic partner, both of conformal weight 0 , so that $h_{\min }=0$. The effective central charge in this sector is therefore $c_{\text {eff }}=-2$, and the general formula (6) reproduces the finite-size corrections (2).

When $M$ is odd and $N$ is even, dimer coverings exist without the need to remove a corner. In this case, the above construction leads to a set of spanning trees on the odd-odd sublattice $\mathcal{G}$, where certain arrows may point out of the lattice from the right vertical side, see Fig. 3. Viewing this vertical boundary of $\mathcal{G}$ as roots for the spanning trees, we see that dimer coverings on $\mathcal{L}$ map onto spanning trees on $\mathcal{G}$ which can grow from any site of the right side. The sites on this boundary, being connected to roots, are dissipative in the ASM language, and form an open boundary. Thus the spanning trees map onto the ASM configurations with one vertical open, dissipative boundary, and the three other closed.

In the limit $M \rightarrow \infty$, the lattice becomes an infinite strip with open and closed boundary conditions on the two sides. In this case, the ground-state of the Hamiltonian with such boundary conditions is a primary field of conformal weight $h_{\min }=-1 / 8$ [33]. With $c=-2$, this yields $c_{\text {eff }}=1$ and again the formula (6) gives the correct result (3).

Thus the leading finite-size corrections for an infinitely long strip of width $N$ agree with the prediction of a $c=-2$ conformal field theory, provided one realizes that changing the parity of $N$ genuinely changes the boundary conditions, an effect due to the strong non-locality of the 

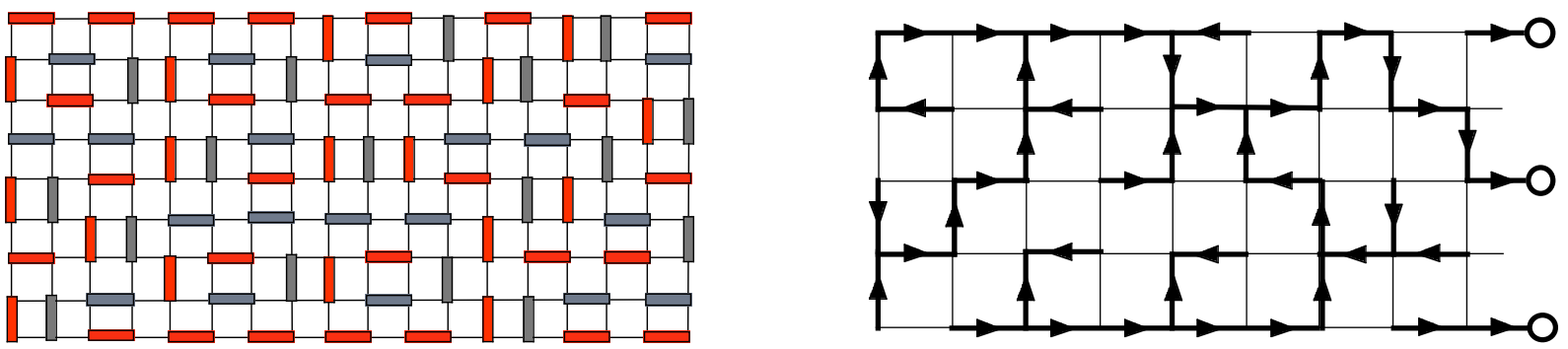

Figure 3. The same dimer covering of a $9 \times 18$ lattice as in Fig. 1 is shown on the left, where the dimers which touch the odd-odd sublattice $\mathcal{G}$ are coloured in red. The right figure shows the corresponding spanning tree on the $5 \times 9$ lattice $\mathcal{G}$; the sites on the right boundary are all connected to roots (the open dots), although in this particular instance, two of these connections have not been used by the tree.
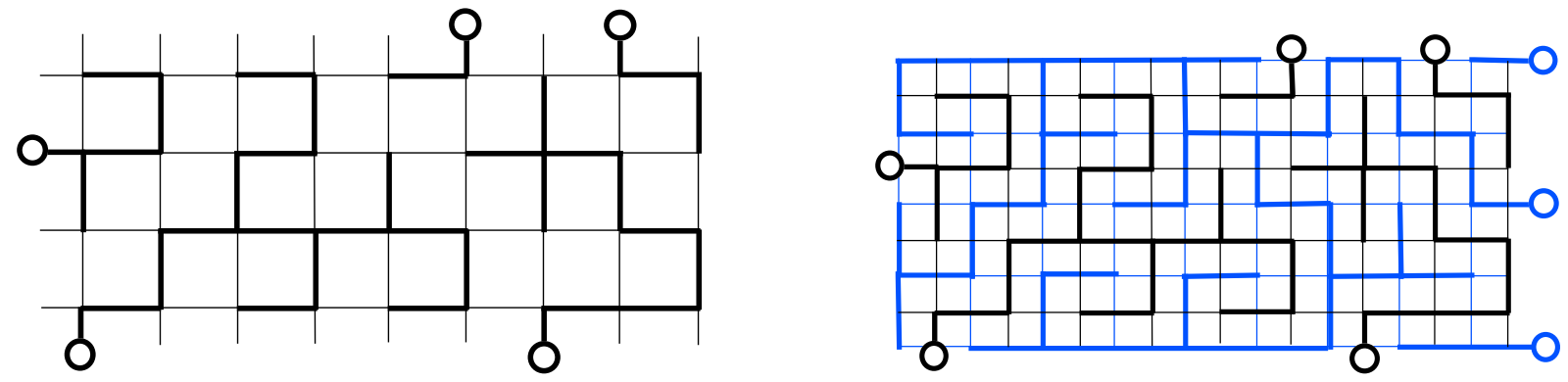

Figure 4. (a) The left figure shows the spanning tree associated to the dimer covering of Fig. 3, drawn on the even-even sublattice rather than on the odd-odd sublattice (the arrows are no longer shown). All boundaries are open (their sites are connected to roots) except the right boundary, which is closed.

(b) The same spanning tree is reproduced on the right figure, where in addition the spanning tree drawn on the odd-odd sublattice, in blue, is superimposed. The duality of the two spanning trees is then manifest.

dimer model [21]. The change of boundary conditions is not apparent in the dimer model itself, but is manifest when one maps it onto the spanning tree model or the sandpile model.

Interestingly the bijection between the dimer coverings and the spanning trees holds if we use the even-even, even-odd or odd-even sublattice. The boundary conditions however change, but one can easily see what the changes are. If, instead of choosing the odd-odd sublattice, one selects the sites whose horizontal (resp. vertical) coordinates are even, the left and right (resp. bottom and top) boundaries change from closed to open, and from open to closed. So if $N$ is odd, the vertical sides become open rather than closed. The spectrum of the corresponding Hamiltonian changes, with a non-degenerate ground-state being the identity operator [33], so that the value $h_{\min }=0$ remains. If $N$ is even, the left and right boundaries, previously closed and open respectively, become open and closed respectively, so the Hamiltonian remains the same, $h_{\min }=-1 / 8$. Fig. 4 shows the spanning tree associated to the dimer covering in Fig. 3 if we choose the even-even sublattice.

In fact the odd-odd and even-even sublattices are dual, as are the corresponding spanning trees, see Fig. 4b. The same is true of the odd-even and even-odd sublattices. Under this duality, the boundary conditions open and closed are exchanged.

\section{Dimers on a cylinder}

We consider here an $M \times N$ rectangular lattice $\mathcal{L}$ with periodic boundary condition in the horizontal direction, so that $\mathcal{L}$ is a cylinder of perimeter $N$ and height $M$. As before, we will 
eventually take $M$ to infinity, which makes its parity irrelevant. For convenience we choose $M$ even. We discuss the cases $N$ odd and $N$ even separately.

If $N$ is odd, we select again the odd-odd sublattice $\mathcal{G}$. It is easy to see that two columns of $\mathcal{G}$ will be neighbours in $\mathcal{G}$ and in $\mathcal{L}$ (connected by horizontal bonds). Therefore a dimer may cover zero, one or two sites of $\mathcal{G}$. As before the dimers covering no site of $\mathcal{G}$ are completely fixed by the others and play no role. For the others, we do the same construction as in the previous section. If a dimer touches only one site of $\mathcal{G}$, we draw an arrow directed along the dimer from that site to the nearest neighbouring site of $\mathcal{G}$. However, for a dimer laid on two sites of $\mathcal{G}$, the two arrows would point from either site to the other, ruining the spanning tree picture. It can nevertheless be restored in the following way.

Instead of seeing the two arrows as pointing from one site to its neighbour, we say that they point towards roots inserted between the neighbour sites, thus replacing the arrows $\bullet \longrightarrow \bullet$ by $\bullet \rightarrow \circ \leftrightarrow$. This in effect amounts to opening the cylinder by removing the horizontal bonds of $\mathcal{L}$ which connect sites of $\mathcal{G}$, unwrapping it into a strip, and to adding columns of roots on the left and on the right side of the strip. The new arrow configurations define spanning trees, rooted anywhere on the left and right boundaries. So dimer coverings on the original cylinder are mapped to spanning trees on a strip, with open upper and closed lower horizontal boundaries (by the arguments of the previous section, since we chose $M$ even), and open vertical boundaries.

However these two vertical boundaries are not independent. If a tree grows from a site $i$ on the left boundary, then a tree must grow from the site $j$ on the right boundary, where $j$ is the site which were neighbour of $i$ in $\mathcal{G}$ before the opening of the cylinder. Conversely if no tree grows from $i$, then no tree can grow from $j$. Thus the growth patterns on the left and on the right boundaries must exactly match.

For finite $N$, this correlation between the two boundaries will be felt throughout the lattice. As $N$ (and $M$ ) increase, the generic properties of the spanning trees will not change if the trees are allowed to grow from slightly different boundary sites. Thus if the density of roots on the left and on the right boundaries is finite, it will make no difference whether the tree growth patterns are identical or random on each boundary.

Following this argument, when $M$ goes to infinity, the lattice becomes an infinite strip with open boundary condition on either side. As mentioned above, the ground-state of the Hamiltonian is the identity, of weight $h_{\min }=0$, leading to an effective central charge $c_{\text {eff }}=-2$. The general formula (6) for the strip gives the correct result (4).

This is a very unusual situation. Although the dimer model is originally defined on a cylinder, it shows the finite-size corrections expected on a strip, and must really be viewed as a model on a strip.

For $N$ even, the problem of having a dimer occupying two neighbouring sites of $\mathcal{G}$ does not arise. Hence the arrows point from one site of $\mathcal{G}$ to a neighbouring site of $\mathcal{G}$ and they never overlap. However the arrow configurations one obtains do not define spanning trees because there can be sequences of arrows looping around the cylinder. In general the arrows form a combination of oriented loops wrapped around the cylinder with trees growing from the loops. The one-to-one correspondence between the oriented loops combined with tree branches on one side, and dimer configurations on the other side can be established as above. The enumeration of the loop-tree configurations requires a slight generalization of Kirchhoff's theorem. In order to appreciate it, we make a little digression, to recall the combinatorial content of the usual Kirchhoff theorem (see for instance [27]), as used in Section 3.

Define the Laplacian of an unoriented graph $\mathcal{G}$ as the symmetric matrix $\Delta$ with entries $\Delta_{i, j}=-1$ if there is a bond connecting site $i$ and site $j \neq i$, and $z_{i}=\Delta_{i, i}$ equal to the number of sites to which $i$ is connected, including the $\operatorname{root}(\mathrm{s})$. Thus $\sum_{j} \Delta_{i, j}$ is the number of connections from $i$ to the $\operatorname{root}(\mathrm{s})$. Let us now place, at each site $i$ of $\mathcal{G}$, an arrow pointing to any one of the $z_{i}$ neighbours of $i$ (possibly to the root if $i$ has a connection to it). Such a set of arrows defines 
an arrow configuration on $\mathcal{G}$, and we want to show that the determinant of $\Delta$ precisely counts those arrow configurations which contain no loop.

The determinant is a sum over the permutations in $S_{N}$ with $N=|\mathcal{G}|$ the number of sites of $\mathcal{G}$,

$$
\operatorname{det} \Delta=\sum_{\sigma \in S_{N}} \varepsilon_{\sigma} \Delta_{1, \sigma(1)} \Delta_{2, \sigma(2)} \cdots \Delta_{N, \sigma(N)} .
$$

Writing a permutation as a product of cycles of lengths $\ell_{m}$, and using $\varepsilon_{\sigma}=(-1)^{N+\# \text { cycles }}$, it is

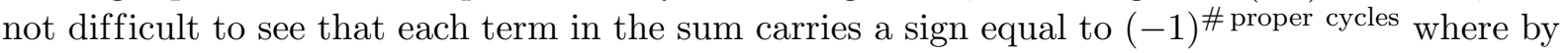
proper cycle we mean a cycle of length strictly larger than 1. Up to this sign, a permutation $\sigma$ with exactly $k$ proper cycles brings a contribution equal to $\prod_{i} z_{i}$ where the product is over those sites left invariant by the permutation. Combinatorially this number counts the arrangements of arrows containing $k$ loops whose locations in the graph are completely specified by the cycle structure of $\sigma$; on the other hand, the arrows coming out of the sites fixed by $\sigma$ are free to point in any of the allowed directions, and may therefore form themselves other loops.

The sum over the permutations can now be reorganized as a sum over the number $k$ of proper cycles,

$$
\operatorname{det} \Delta=\sum_{k=0}^{[N / 2]}(-1)^{k} \sum_{\sigma \text { has } k \text { proper cycles }}\left|\Delta_{1, \sigma(1)} \cdots \Delta_{N, \sigma(N)}\right| .
$$

The term for $k=0$, equal to $\prod_{i \in \mathcal{G}} z_{i}$ counts the total number of unconstrained arrow configurations. The term $k=1$, up to the minus sign, counts the arrow configurations which have at least one loop of a fixed type, and sums over the possible loops. It therefore overcounts the number of arrow configurations with at least one loop by the number of arrow configurations which have at least two loops. This is taken care of by the $k=2$ term, which however overcounts it by a amount equal to the number of configurations with at least three loops, and so on. By the inclusion-exclusion principle, the alternating sum (8) exactly counts the configurations of arrows with no loop at all.

Let us now come back to the problem of counting the loop-tree configurations of arrows, in which the only loops we allow must wrap around the cylinder (i.e. be non-contractible). Because they cannot intersect themselves, the loops can wrap only once around the cylinder. For this, we modify the previous Laplacian $\Delta$ by changing from -1 to +1 the entries $\Delta_{i, j}$ for all pairs $i, j$ such that the bond $i-j$ crosses a line going from one boundary of the cylinder to the other boundary, which we will call a defect line. The resulting still symmetric matrix $\Delta_{A}$ can be viewed as the antiperiodic Laplacian (the lower and upper boundaries are closed and open, or vice-versa, since we have taken $M$ even). We show that $Z=\operatorname{det} \Delta_{A}$ precisely counts the loop-tree configurations we need.

The key observation is that a contractible loop on the cylinder crosses the defect line an even number of times, whereas a non-contractible loop crosses it an odd number of times. As a consequence, and with respect to the previous situation, there is an extra minus sign for each proper cycle giving rise to a non-contractible loop of arrows. One thus obtain

$$
\operatorname{det} \Delta_{A}=\sum_{k=0}^{[N / 2]}(-1)^{k} \sum_{\sigma \text { has } k \text { proper cycles }}(-1)^{\# \text { non-contr. }}\left|\Delta_{1, \sigma(1)} \cdots \Delta_{N, \sigma(N)}\right| .
$$

If we write $k=p+q$, where $p$ is the number of non-contractible loops (NCL) and $q$ is the number of contractible loops (CL), the summation over $k$ is replaced by two summations over $p$ and $q$,

$$
\operatorname{det} \Delta_{A}=\sum_{p \geq 0} \sum_{q \geq 0}(-1)^{q} \sum_{\sigma \text { has } p \text { NCL }, q \text { CL }}\left|\Delta_{1, \sigma(1)} \cdots \Delta_{N, \sigma(N)}\right| .
$$


One sees that the arrow configurations with a fixed number $p$ of non-contractible loops, i.e. the terms with $q=0$, are all counted positively. Moreover, for each $p$, the inner summation over $q$ is an alternating sum which implements the inclusion-exclusion principle and removes all contractible loops. Therefore $\operatorname{det} \Delta_{A}$ exactly counts the arrow configurations with no contractible loops, as claimed.

In the continuum limit, it becomes the partition function of a free theory of antiperiodic Grassmannian fields which, in turn, gives $c=-2$ and $h_{\min }=-1 / 8$ [33]. This is again consistent, since the finite size correction (4) together with the general formula (7) for the cylinder yield $c_{\text {eff }}=1$.

\section{Conclusion}

By analyzing the finite-size effects in terms of the effective central charge $c_{\text {eff }}=c-24 h_{\min }$, we have shown that the non-local boundary effects in the close-packed dimer model can be consistently accounted for by a single conformal theory having central charge $c=-2$. We have provided a consistent framework for understanding the dependence of the finite-size effects upon the boundary conditions. However this should not be taken as a proof that $c$ must be equal to -2 . Indeed since the effective central charge merely determines some combination of $c$ and $h_{\text {min }}$, one cannot obtain the values of both without some assumption about one of them. This assumption can be a posteriori justified if the conformal description obtained from it is fully consistent.

It turns out in this case that another consistent conformal description exists, with $c=1$ $[23,24]$, although a detailed analysis of boundary conditions and parity dependence effects has not been carried out in this context. Our explanation for this curious fact is that the $c=1$ theory not only describes the close-packed dimer model, but the general monomer-dimer model (it has been suggested in [37] that trimers would require $c=2$ ).

On one hand, this has been illustrated for instance in [8] where the monomer 2-point correlation function [6] has been interpreted in terms of two uncoupled Majorana fermions. The same interpretation can be made for the general $n$-point function for monomers on a boundary [38]. On the other hand, the spanning tree description leading to the value $c=-2$ cannot describe dimers with monomers in generic positions, for the basic reason that they are defined on a sublattice and therefore cannot keep track of the positions of all the monomers.

Thus the conformal theory with $c=-2$ must be viewed as a subtheory of that with $c=1$, as it is able to describe the degrees of freedom corresponding to dimer coverings but not general monomer insertions. For those degrees of freedom, the two descriptions should be equivalent.

\section{Acknowledgements}

P.R. is financially supported by the Belgian Fonds National de la Recherche Scientifique (FNRS).

\section{References}

[1] Fowler R.H., Rushbrooke G.S., Statistical theory of perfect solutions, Trans. Faraday Soc. 33 (1937), 12721294.

[2] Kasteleyn P.W., The statistics of dimers on a lattice. I. The number of dimer arrangements on a quadratic lattice, Physica 27 (1961), 1209-1225.

[3] Kasteleyn P.W., Dimer statistics and phase transitions, J. Math. Phys. 4 (1963), 287-293.

[4] Fisher M.E., Statistical mechanics of dimers on a plane lattice, Phys. Rev. 124 (1961), 1664-1672.

[5] Temperley H.N.V., Fisher M.E., Dimer problem in statistical mechanics - an exact result, Philos. Mag. (8) 6 (1961), 1061-1063. 
[6] Fisher M.E., Stephenson J., Statistical mechanics of dimers on a plane lattice. II. Dimer correlations and monomers, Phys. Rev. 132 (1963), 1411-1431.

[7] Hartwig R.E., Monomer pair correlations, J. Math. Phys. 7 (1966), 286-299.

[8] Fendley P., Moessner R., Sondhi S.L., Classical dimers on the triangular lattice, Phys. Rev. B 66 (2002), 214513, 14 pages, cond-mat/0206159.

[9] Basor E.L., Ehrhardt T., Asymptotics of block Toeplitz determinants and the classical dimer model, math-ph/0607065.

[10] Tseng W.J., Wu F.Y., Dimers on a simple-quartic net with a vacancy, J. Statist. Phys. 110 (2003), 671-689.

[11] Kong Y., Logarithmic corrections in the free energy of monomer-dimer model on plane lattices with free boundaries, Phys. Rev. E $\mathbf{7 4}$ (2006), 011102, 8 pages.

[12] Wu F.Y., Pfaffian solution of a dimer-monomer problem: single monomer on the boundary, Phys. Rev. E 74 (2006), 020140(R), 4 pages, Erratum, Phys. Rev. E 74 (2006), 020104(E), cond-mat/0607647.

[13] Ferdinand A.E., Statistical mechanics of dimers on a quadratic lattice, J. Math. Phys. 8 (1967), 2332-2339.

[14] McCoy B.W., Wu T.T., The two-dimensional Ising model, Harvard University Press, Cambridge, MA, 1973.

[15] Bhattacharjee S.M., Nagle F.F., Finite-size effect for the critical point of an anisotropic dimer model of domain walls, Phys. Rev. A 31 (1985), 3199-3213.

[16] Brankov J.G., Priezzhev V.B., Critical free energy of a Möbius strip, Nuclear Phys. B 400 (1993), 633-652.

[17] Lu W.T., Wu F.Y., Dimer statistics on the Möbius strip and the Klein bottle, Phys. Lett. A 259 (1999), 108-114, cond-mat/9906154.

[18] Lu W.T., Wu F.Y., Close-packed dimers on nonorientable surfaces, Phys. Lett. A 293 (2002), 235-246, Erratum, Phys. Lett. A 298 (2002), 293, cond-mat/0110035.

[19] Ivashkevich E., Izmailian N.Sh., Hu C.-K., Kronecker's double series and exact asymptotic expansion for free models of statistical mechanics on torus, J. Phys. A: Math. Gen. 35 (2002), 5543-5561.

[20] Izmailian N.Sh., Oganesyan K.B., Hu C.-K., Exact finite-size corrections of the free energy for the square lattice dimer model under different boundary conditions, Phys. Rev. E 67 (2003), 066114, 14 pages.

[21] Izmailian N.Sh., Priezzhev V.B., Ruelle P., Hu C.-K., Logarithmic conformal field theory and boundary effects in the dimer model, Phys. Rev. Lett. 95 (2005), 260602, 4 pages, cond-mat/0512703.

[22] Itzykson C., Saleur H., Zuber J.-B., Conformal invariance of nonunitary 2d-models, Europhys. Lett. 2 (1986), 91-96.

[23] Blote H.W.J., Hilhorst H.J., Roughening transitions and the zero-temperature triangular Ising antiferromagent, J. Phys. A: Math. Gen. 15 (1982), L631-L637.

[24] Kenyon R., Dominos and the Gaussian free field, Ann. Probab. 29 (2001), 1128-1137.

[25] Baxter R.J., Exactly solved models in statistical mechanics, Academic Press, New York, 1982.

[26] Temperley H.N.V., Combinatorics, in Proceedings of the British Combinatorial Conference, London Math. Soc. Lecture Notes Series 13 (1974), 202-204.

[27] Priezzhev V.B., The dimer problem and the Kirchhoff theorem, Sov. Phys. Usp. 28 (1985), $1125-1135$.

[28] Blöte H.W., Cardy J.L., Nightingale M.P., Conformal invariance, the central charge, and universal finite-size amplitudes at criticality, Phys. Rev. Lett. 56 (1986), 742-745.

[29] Affleck I., Universal term in the free energy at a critical point and the conformal anomaly, Phys. Rev. Lett. 56 (1986), 746-748.

[30] Cardy J.L., Effect of boundary conditions on the operator content of two-dimensional conformally invariant theories, Nuclear Phys. B 275 (1986), 200-218.

[31] Brankov J.G., Isomorphism of dimer configurations and spanning trees on finite square lattices, J. Math. Phys. 36 (1995), 5071-5083.

[32] Majumdar S.N., Dhar D., Equivalence between the Abelian sandpile model and the $q \rightarrow 0$ limit of the Potts model, Phys. A 185 (1992), 129-145.

[33] Ruelle P., A $c=-2$ boundary changing operator for the Abelian sandpile model, Phys. Lett. B 539 (2002), 172-177, hep-th/0203105.

[34] Piroux G., Ruelle P., Pre-logarithmic and logarithmic fields in a sandpile model, J. Stat. Mech. Theory Exp. (2004), P10005, 24 pages, hep-th/0407143. 
[35] Piroux G., Ruelle P., Logarithmic scaling for height variables in the Abelian sandpile model, Phys. Lett. B 607 (2005), 188-196, cond-mat/0410253.

[36] Jeng M., Piroux G., Ruelle P., Height variables in the Abelian sandpile model: scaling fields and correlations, J. Stat. Mech. Theory Exp. (2006), P10015, 63 pages, cond-mat/0609284.

[37] Ghosh A., Dhar D., Jacobsen J.L., Random trimer tilings, cond-mat/0609322.

[38] Priezzhev V.B., Ruelle P., Boundary monomers in the dimer model, in preparation. 